\title{
AN AXIOMATIC APPROACH TO THE BOUNDARY THEORIES OF WIENER AND ROYDEN
}

\author{
BY PETER A. LOEB AND BERTRAM WALSH ${ }^{1}$ \\ Communicated by John W. Green, April 8, 1968
}

In this note we announce results, obtained in the framework of Brelot's axiomatic potential theory, which are applicable to the Wiener and Royden boundary theories for Riemann surfaces. ${ }^{2}$ Recall that in Brelot's theory, we consider a sheaf $\mathfrak{F C}$ of real-valued functions with open domains contained in a locally compact, noncompact, connected and locally connected Hausdorff space $W$, with the functions satisfying certain axioms. Specifically, by a harmonic class of functions on $W$ we mean a class $\mathcal{H}$ of real-valued continuous functions with open domains. For each open $\Omega \subseteq W, \mathcal{F}_{\Omega}$ denotes the set of functions in $\mathcal{H}$ with domains equal to $\Omega$; it is assumed that $\mathcal{H}_{\Omega}$ is a real vector space. The three axioms of Brelot which $\mathfrak{F C}$ is assumed to satisfy are (1) a function is in $\mathfrak{H C}$ if and only if it is locally in $\mathfrak{H C}$; (2) there is a base for the topology of $W$ which consists of regions regular for $\mathfrak{H}$, i.e. connected open sets $\omega$ such that any continuous function $f$ on $\partial \omega$ has a unique continuous extension in $\mathcal{F}_{\omega}$ which is nonnegative if $f$ is nonnegative; (3) the upper envelope of any increasing sequence of functions in $\mathcal{F C}_{\Omega}$ where $\Omega$ is a region (i.e. open and connected) is either $+\infty$ or an element of $\mathcal{H}_{\Omega}$.

Let $\mathfrak{F C}^{-}$and $\mathfrak{H C}_{-}$denote the classes of functions which are superharmonic and subharmonic with respect to $\mathfrak{K}$; let $\mathfrak{H}^{-b}$ denote the subclass of $\mathrm{FC}^{-}$consisting of functions bounded below. We assume as another axiom: (4) $1 \in \mathfrak{H}_{\bar{W}}$.

1. Let $\bar{W}$ be a Hausdorff space in which $W$ is imbedded as a dense (and therefore open) subspace, and henceforth let us agree that $\bar{\Omega}$ will mean the closure of $\Omega$ in $\bar{W}$ and $\partial \Omega=\Omega-\Omega$. If $\Omega$ is an open subset of $W$, we shall say that $\partial \Omega$ is associated with $\mathfrak{F C}_{\Omega}^{-b}$ if every $v \in \mathfrak{F C}_{\Omega}^{-b}$ whose limit inferior is nonnegative at every point of $\partial \Omega$ is necessarily nonnegative on $\Omega$. Throughout this note, we shall denote $\lim _{x \in \Omega, x \rightarrow x_{0}} f(x)$ by $\lim _{\Omega} f\left(x_{0}\right)$; similar notation is used for lim inf and lim sup.

THEOREM 1.1. If $\Omega$ is an open subset of $W$ and $\partial W$ is associated with $\mathfrak{H C}_{W}^{-b}$, then $\partial \Omega$ is associated with $\mathcal{H C}_{\Omega}^{-b}$.

1 The first author was supported by National Science Foundation research grants GP-5279 and GP-4653; the second author by GP-4563.

2 These results will appear with proofs as part of a forthcoming article in the Annales de l'Institut Fourier. 
Assume that $\partial W$ is associated with $\mathcal{F}_{W}^{-b}$; then given a bounded realvalued function $f$ on $\partial \Omega$ (where $\Omega$ is an open subset of $W$ ) one can define $H^{-}(f, \Omega) \in \mathcal{F C}$ to be the lower envelope of the set $\left\{v \in \mathcal{F}_{\Omega}^{-b}\right.$ : lim $\inf _{\Omega} v(x) \geqq f(x)$ for all $\left.x \in \partial \Omega\right\}$ and dually define $H_{-}(f, \Omega)$ $=-H^{-}(-f, \Omega) . H^{-}(f, \Omega)$ and $H_{-}(f, \Omega)$ are respectively the upperand lower- $\mathfrak{H}$-extensions of $f$ in $\Omega$. If they are equal, we say that $f$ is resolutive on $\partial \Omega$. A point $x_{0} \in \partial \Omega$ for which $\lim \sup H^{-}(f, \Omega)\left(x_{0}\right)$ $\leqq \lim \sup f\left(x_{0}\right)$ for every bounded function $f$ on $\partial \Omega$ is said to be regular (with respect to $\mathfrak{F}$ ). Given $x_{0} \in \partial \Omega$, a positive function $b \in \mathfrak{H}^{-}$defined in the intersection of $\Omega$ with an open neighborhood of $x_{0}$ and for which $\lim _{\Omega} b\left(x_{0}\right)=0$ is called an Fe-barrier (or simply a barrier) for $\Omega$ at $x_{0}$. We say that there is a system of barriers for $\Omega$ (or, for emphasis, $\bar{\Omega}$ ) at $x_{0}$ if there is a base $\theta$ for the neighborhood system of $x_{0}$ such that on the intersection of $\Omega$ with $\omega \in \mathcal{O}$ there is defined a barrier $b$ for $\Omega$ at $x_{0}$ with

$$
\inf \left\{\lim \inf _{\Omega} b\left(x_{1}\right): x_{1} \in \partial(\omega \cap \Omega)-(\omega \cap \partial \Omega)\right\}>0 .
$$

Such a barrier is said to belong to $\Omega$ and $\omega$. An $\mathcal{H}_{-}$-unit-barrier for $\Omega$ at $x_{0}$ is a function $b_{1} \in \mathcal{F}_{-}$, defined on the intersection of $\Omega$ with a neighborhood of $x_{0}$ and such that $\lim _{\Omega} b_{1}\left(x_{0}\right)=1$. With these definitions, we have

TheOREM 1.2. Let $x_{0}$ be a point of $\partial \Omega$. Assume there is a system of barriers and an $H_{-}-$unit-barrier for $\Omega$ at $x_{0}$. Then $x_{0}$ is a regular point for $\Omega$.

2. Let $\mathfrak{H}$ be a harmonic class which is hyperbolic on $W$ [5, p. 189], and let $\mathrm{BHC}_{W}$ denote the set of all bounded $\mathcal{H}$-harmonic functions on $W$. Then $B^{B} C_{W}$ is a Banach lattice with order unit $H(W)$, where $H(W)$ is the greatest $\mathcal{H C}$-harmonic minorant of 1 . The lattice operation $V_{\mathcal{F C}}$ is given by defining $f \bigvee_{\mathcal{F C}}$ to be the least $\mathfrak{F C}$-harmonic majorant of the pointwise supremum $f \vee g$, and $\wedge_{\mathcal{F C}}$ is similarly defined.

We next consider ideal boundary theory for an arbitrary Banach sublattice $\mathfrak{S}$ of $\mathbb{B H C}_{W}$ when $H(W) \in \mathfrak{S}$. Some examples of such sublattices are:

(1) ${ }^{\circ} \mathcal{H}_{W}$ itself.

(2) The uniform closure of the space $B D \mathfrak{H}_{W}$, where $B D \mathcal{H}_{W}$ is the set of all bounded harmonic functions (in the usual sense) with finite Dirichlet in tegral on an open Riemann surface $W$.

(3) The uniform closure of the space of all bounded $C^{2}$-functions $f$ on an open Riemann surface $W$ such that:

(a) $\Delta f=P f$ where $P$ is a nonnegative density on $W$ with $\iint_{W} P<\infty$, and 
(b) $D(f, f)+\iint_{W} P f^{2}<\infty$ where $D(f, f)$ is the Dirichlet integral of $f$.

Let a Banach sublattice $\mathfrak{S}$ of $\mathcal{B F}_{W}$ containing the order unit, $H(W)$, be given. Now form the $Q$-compactification [2, pp. 96-97] and [6] $W_{\mathfrak{W}}^{*}$ of $W$ with $Q=\mathfrak{S}$; this is a compact Hausdorff space containing $W$ as a dense subspace, determined up to homeomorphism by the properties that each $f \in \mathfrak{S}$ has a continuous extension to $W_{\mathfrak{S}}^{*}$ and that the family of all these extensions separates the points of $\Delta_{\mathfrak{Q}}=W_{\mathfrak{Q}}^{*}-W$. Define

$$
\Gamma_{\mathfrak{G}}=\left\{t \in \Delta_{\mathfrak{G}}: H(W)(t)=1\right\} \cap \bigcap_{f, g \in \mathfrak{W}}\left\{t \in \Delta_{\mathfrak{G}}:\left(f \Lambda_{\mathfrak{K} g}\right)(t)=(f \wedge g)(t)\right\}
$$

and let $\bar{W}_{\mathfrak{s}}=W \cup \Gamma_{\mathfrak{s}}$. Then

THEOREM 2.1. $\Gamma_{\mathfrak{S}}$ is associated with $\mathfrak{F}_{W}^{-b}$, whence $\Gamma_{\mathfrak{S}}$ is nonempty.

THEOREM 2.2. If $M \subseteq \Delta_{\mathfrak{G}}$ is a closed set which is associated with $\mathfrak{H}_{W}^{-b}$, then the restriction map $f \rightarrow f \mid M$ of $\mathfrak{S}_{\text {into }} \mathfrak{C}_{R}(M)$ is an isometry (not necessarily onto) preserving positivity in both directions.

Now by the lattice form of the Stone-Weierstrass theorem we have

THEOREM 2.3. The restriction mapping $f \rightarrow f \mid \Gamma_{\mathfrak{S}}$ of $\mathfrak{S}$ into $\mathfrak{C}_{R}\left(\Gamma_{\mathfrak{S}}\right)$ is a surjective isometry sending the order unit of $\mathfrak{S}$ to the order unit 1 of $\mathfrak{C}_{R}\left(\Gamma_{\mathfrak{W}}\right)$ and preserving the lattice operations.

THEOREM 2.4. $\Gamma_{\mathfrak{g}}$ is the intersection of all sets $\Gamma_{p}=\left\{t \in \Delta_{\mathfrak{g}}\right.$ : $\lim \inf p(t)=0\}$ as $p$ ranges through the $\mathfrak{F}$-potentials on $W$. No proper closed subset of $\Gamma_{\mathfrak{S}}$ is associated with $\mathfrak{H}_{W}^{-b}$.

THEOREM 2.5. Except perhaps when $\mathfrak{S}$ consists only of constant functions, there is an $\mathfrak{H}_{-}-$unit barrier and a system of barriers for $W_{\mathfrak{5}}^{*}$ at each point of $\Gamma_{\mathfrak{5}}$, whence each $x \in \Gamma_{\mathfrak{S}}$ is regular with respect to any open set $\Omega \subset W$ for which $x \in \partial \Omega \cap \Gamma_{\mathfrak{g}}$. (Here $\partial \Omega$ is taken in $W_{\mathfrak{\$}}^{*}$.)

THEOREM 2.6. Let $\tilde{\mathfrak{S}}$ denote those bounded functions in $\mathfrak{H}_{W}^{-}$for which the greatest $\mathfrak{H}$-harmonic minorant is in $\mathfrak{S}$. For any $v \in \tilde{\mathfrak{H}}$, let $I(v)$ be the function on $\Gamma_{\mathfrak{g}}$ defined by $I(v)(t)=\lim \inf _{W} v(t)$ for each $t \in \Gamma_{\mathfrak{S}}$. Then $I(v)$ is continuous on $\Gamma_{\mathfrak{g}}$ for each $v \in \tilde{\mathfrak{S}}$, and the mapping $I: \tilde{\mathfrak{h}} \rightarrow \mathfrak{C}_{R}\left(\Gamma_{\mathfrak{\xi}}\right)$ is positively homogeneous and additive.

If $W$ is an open Riemann surface, $\mathfrak{H C}$ the class of harmonic functions in the usual sense, and $\mathfrak{S}=\mathcal{B F}_{W}$, then $\Gamma_{\mathfrak{g}}$ is homeomorphic to the harmonic part of the Wiener boundary even though $\Delta_{\mathfrak{S}}$ is "smaller" than the Wiener boundary. If $\mathfrak{S}_{\mathfrak{E}}$ is the uniform closure of $B D \mathcal{H}_{W}$, the bounded harmonic functions with finite Dirichlet integrals, then $\Gamma_{\mathfrak{G}}$ 
is the harmonic part of the Royden boundary and $B D \mathcal{H}_{W}$ is isometrically isomorphic to a dense subset of $\mathfrak{C}_{R}\left(\Gamma_{\mathfrak{g}}\right)$.

\section{BIBLIOGRAPHY}

1. M. Brelot, Lectures on potential theory, Tata Institute of Fundamental Research, Bombay, 1960.

2. C. Constantinescu and A. Cornea, Ideale Ränder Riemannscher Flächen, Ergebnisse Math. (2) 32 (1963).

3. - Compactifications of harmonic spaces, Nagoya Math. J. 25 (1965), 1-57.

4. S. Kakutani, Concrete representation of abstract (M)-spaces, Ann. of Math. (2) 42 (1941), 994-1024.

5. P. A. Loeb, An axiomatic treatment of pairs of elliptic differential equations, Ann. Inst. Fourier (Grenoble) 16 (1966), 167-208.

6. - A minimal compactification for extending continuous functions, Proc. Amer. Math. Soc. 18 (1967), 282-283.

7. P. A. Loeb and B. Walsh, The equivalence of Harnack's principle and Harnack's inequality in the axiomatic system of Brelot, Ann. Inst. Fourier (Grenoble) 15 (1965), $597-600$.

8. B. Walsh and P. A. Loeb, Nuclearity in axiomatic potential theory, Bull. Amer. Math. Soc. 72 (1966), 685-689.

University of California, Los Angeles 